\title{
Aharonov-Bohm oscillations in the magnetic moment of multielectron randomly doped semiconductor cylindrical core-shell nanowires
}

\author{
V. N. Gladilin, J. Tempere, and J. T. Devreese \\ TQC-Theory of Quantum and Complex Systems, Universiteit Antwerpen, Universiteitsplein 1, B-2610 Antwerpen, Belgium \\ P. M. Koenraad \\ PSN-Photonics and Semiconductor Nanophysics, COBRA, Eindhoven University of Technology, \\ P.O. Box 513, NL-5600 MB Eindhoven, The Netherlands \\ (Received 2 January 2013; revised manuscript received 27 March 2013; published 15 April 2013)
}

\begin{abstract}
Energy spectra and the magnetic moments of semiconductor core-shell nanocylinders, subjected to a magnetic field, are theoretically studied for different numbers of electrons in the shell and different height-to-radius ratios of the cylinder. The electron-electron interaction is taken into account within the Hartree-Fock approximation. We focus on the perspectives to experimentally detect the Aharonov-Bohm oscillations in the magnetic moment of multielectron core-shell nanowires. Among the different factors that influence the shape and magnitude of these oscillations, the destructive effect due to the interaction of electrons with randomly distributed charged impurities appears most important. Nevertheless, Aharonov-Bohm oscillations of a sufficiently high magnitude survive when averaged over an assembly of nanowires with different impurity distributions.
\end{abstract}

DOI: 10.1103/PhysRevB.87.165424

PACS number(s): 73.21.Hb, 73.23.Ra, 73.22.Dj

\section{INTRODUCTION}

Electrons confined to a submicron doubly connected structure can manifest a topologically determined quantuminterference phenomenon, known as the Aharonov-Bohm (AB) effect, ${ }^{1}$ as a result of the oscillatory behavior of their energy levels as a function of an applied magnetic field. This behavior is usually associated with the occurrence of oscillatory persistent currents. ${ }^{2-4}$ The existence of persistent currents on macroscopic scales has been considered a hallmark of superconductivity in metals ever since its discovery. In 1983, Büttiker et al. ${ }^{4}$ pointed out that persistent currents should also exist in nonsuperconducting loops threaded by magnetic flux. For nonsuperconducting structures, the experimental evidence of $\mathrm{AB}$ oscillations was found in magnetoresistance measurements on normal-metal mesoscopic (with diameters $\sim 0.4$ to $2 \mu \mathrm{m}$ ) rings $^{5-7}$ and hollow cylinders ${ }^{8}$ as well as in magnetization of metallic ${ }^{9,10}$ and semiconductor ${ }^{11}$ rings.

In the past decade, advances in manufacturing selforganized ringlike semiconductor nanostructures (see, e.g., Refs. 12-17) have stimulated theoretical and experimental studies of the AB oscillations in the persistent currents carried by single- and few-particle states (see, e.g., Refs. 18-24). Using an ultrasensitive torsion magnetometer, $\mathrm{AB}$ oscillations in the magnetic moment of $\operatorname{In}_{x} \mathrm{Ga}_{1-x}$ As self-assembled quantum rings have been observed ${ }^{18}$ with a magnitude of oscillation as large as $60 \%$ to $70 \%$ of the corresponding magnitude in an ideal ring. The $\mathrm{AB}$ oscillations have also been experimentally detected in the excitonic magnetophotoluminescence spectra of single quantum rings, ${ }^{23}$ although the corresponding effect is rather weak and it can become unobservable in the spectra averaged over an ensemble of nonidentical rings. ${ }^{22}$ Also, the successful experimental detection of the $\mathrm{AB}$ effect in the magnetic moment of single-electron nanorings ${ }^{18}$ has appeared to be a rather challenging task because the magnetization signal is extremely weak even for a large assembly of rings. It has been shown that imperfections in the ring geometry such as local potential fluctuations can severely affect the magnitude of the anticipated AB effect in the optical, electronic, and magnetic signals. ${ }^{18,19,21,22,25}$ In this paper, astructure, which can be obtained by semiconductor nanowire growth, is proposed for the observation of the $\mathrm{AB}$ effect in the magnetization. We show that this structure has the potential to overcome the limitations of previously studied semiconductor self-assembled quantum rings. ${ }^{26}$ In the past decade, excellent control has been obtained over the vertical and radial growth of semiconductor nanowire structures by using gold catalyst particles $^{27,28}$ and many examples of core-shell structures have been realized. In core-shell nanowire structures, we propose to form a cylindrical confinement potential (quantum well) that can be populated by electrons coming from a modulationdoped region in the core of the nanowire. In contrast with quantum rings, which can be populated with no more than a few electrons and which are very sensitive to potential imperfections, cylindrical nanoshell structures can contain a much larger number of electrons contributing to the $\mathrm{AB}$ effect. Moreover, at sufficiently high doping and electron concentrations, the carriers in the cylindrical confinement potential are expected to experience a smoother potential allowing a better visibility of the $\mathrm{AB}$ effect.

In this paper, we analyze the energy spectrum and the magnetic moment of multielectron quasi-two-dimensional (quasi-2D) cylindrical nanoshells with different aspect ratios and numbers of electrons. In particular, we address the prospects of detecting the $\mathrm{AB}$ effect in the magnetic moment of nanowires with parameters comparable to those reported in Ref. 29. We show that despite a destructive influence of the interaction between electrons and randomly distributed charged impurities, the $\mathrm{AB}$ oscillations remain well pronounced both in the magnetic response of a single nanocylinder and even after averaging the magnetic moment over an ensemble of nanocylinders with different impurity distributions.

The remaining part of the paper is organized as follows. In Sec. II, the $A B$ oscillations in the energy spectrum and in the magnetic moment are considered in the case of noninteracting electrons in quasi-2D cylindrical shells with 


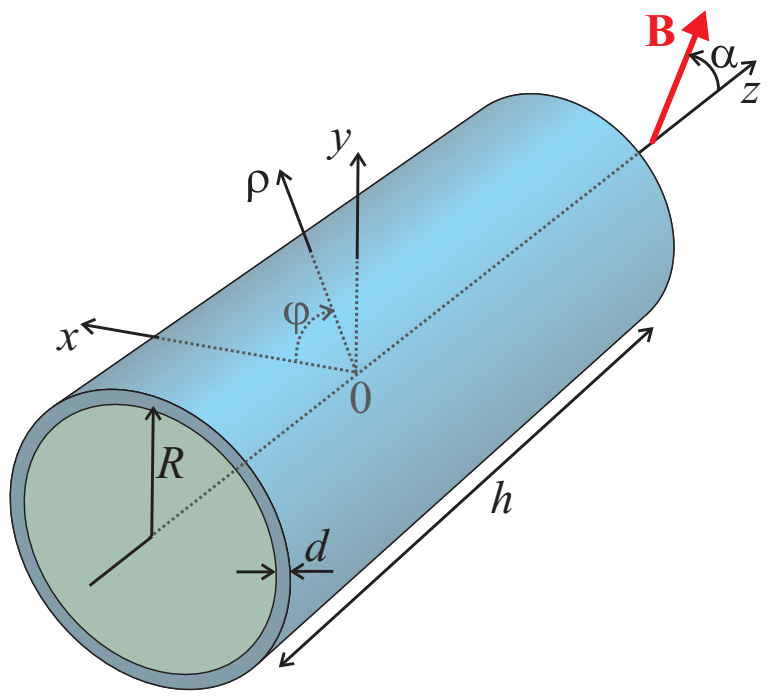

FIG. 1. (Color online) Sketch of the semiconductor core-shell nanocylinder under consideration.

different height-to-radius ratio. We also analyze the experimentally relevant effects of a nonzero shell thickness and of a moderate misalignment of the magnetic field with respect to the cylinder axis. In Sec. III, the model is extended to take into account the electron-electron interaction and the interaction of electrons with charged impurities. The influence of these interactions on the shape of the $\mathrm{AB}$ oscillations of the magnetic moment is discussed for individual multielectron core-shell nanocylinders as well for assemblies of nanocylinders with different distributions of impurities.

\section{AHARONOV-BOHM OSCILLATIONS FOR NONINTERACTING ELECTRONS}

We consider a cylindrical semiconductor core-shell nanostructure, schematically shown in Fig. 1. The height of the cylinder is $h$. The thickness $d$ of the shell is much smaller than its radius $R$. In our calculations, the shell radius is taken within the range from 10 to $15 \mathrm{~nm}$, typical for the structures described in Ref. 29. It is assumed that the material parameters of the core, shell, and the surrounding medium (or a capping layer, which may cover the core-shell structure shown in Fig. 1) provide a deep potential well for electrons within the cylindrical shell. The structure is subjected to a homogeneous magnetic field $\mathbf{B}$, which, in general, can form an angle $\alpha$ with the cylinder axis, chosen to be parallel to the $z$ axis of the used coordinate frame.

In this section, we analyze the situation in which the electrons, confined to the cylindrical shell, interact neither with each other nor with impurities. The single-electron Hamiltonian has the form

$$
H^{(1)}=\frac{\hbar^{2}}{2 m_{e}}\left(-\mathrm{i} \nabla+\frac{e}{\hbar} \mathbf{A}\right)^{2}+\mu_{B} g_{S} B,
$$

where $m_{e}$ is the effective mass of conduction electrons, $e=|e|$ is the elementary charge, $\mathbf{A}$ is the vector potential, the electron $g$ factor is assumed to be $g=2, \mu_{B}$ is the Bohr magneton, and $s$ is the electron spin projection on the $z$ axis. In our calculations below, we set $m_{e} / m_{0}=0.067$ ( $m_{0}$ is the free-electron mass), suitable for GaAs.

\section{A. 2D cylindrical shell in a parallel magnetic field}

In the case where the thickness of the cylindrical shell is negligibly small as compared to its radius, the problem becomes effectively two dimensional. Using cylindrical coordinates $\rho, \varphi, z$ (see Fig. 1) and choosing the gauge for the vector potential as $A_{\rho}=A_{z}=0, A_{\varphi}=B \rho / 2$, so that the magnetic field is parallel to the cylinder axis, the single-electron Schrödinger equation for the coordinate wave functions $\Psi$ becomes

$$
\frac{\hbar^{2}}{2 m_{e}}\left[\left(-\frac{\mathrm{i}}{R} \frac{\partial}{\partial \varphi}+\frac{e B R}{2 \hbar}\right)^{2} \Psi-\frac{\partial^{2} \Psi}{\partial z^{2}}\right]=\epsilon \Psi .
$$

Assuming that the potential barriers for the electrons confined to the shell are infinitely high, Dirichlet boundary conditions can be used for the electron coordinate wave functions $\Psi$. Then, the single-electron wave functions can be written in the form

$$
\Psi_{L n}(\varphi, z)=\frac{1}{\sqrt{2 \pi}} e^{i L \varphi} \chi_{n}(z),
$$

where

$$
\chi_{2 j+1}(z)=\sqrt{\frac{2}{h}} \cos [(2 j+1) \pi z / h], \quad j=0,1,2, \ldots
$$

and

$$
\chi_{2 j}(z)=\sqrt{\frac{2}{h}} \sin (2 j \pi z / h), \quad j=1,2,3, \ldots .
$$

The corresponding single-electron eigenenergies are

$$
E_{L n s}=\frac{\hbar^{2}}{2 m_{e} R^{2}}\left[\left(\frac{\pi n}{\eta}\right)^{2}+\left(L+\frac{\pi R^{2} B}{\Phi_{0}}\right)^{2}\right]+\mu_{B} g s B,
$$

where $\eta=h / R$ is the aspect ratio of the cylinder, $\Phi=\pi R^{2} B$ is the magnetic flux through the cylinder, $\Phi_{0}=2 \pi \hbar / e$ is the flux quantum, and the last term describes the Zeeman splitting of the energy levels.

In Fig. 2, the lowest single-electron energy levels in a cylindrical shell with radius $R=12 \mathrm{~nm}$ and thickness $d \rightarrow 0$ are plotted as a function of the applied magnetic field $B$, parallel to the $z$ axis, for different values of the aspect ratio $\eta$. These plots illustrate the evolution of the energy spectrum from that typical for a (quasi-) $1 \mathrm{D}$ ring at $\eta=0.1$ [see Fig. 2(a)], to the formation of energy "subbands" that correspond to a fixed value of the magnetic quantum number $L$ and different states of quantization along the $z$ axis, in the case of $\eta \gg 1$ [see Fig. 2(d)]. In the case of $\eta=0.1$, the Zeeman contribution to the electron energies is almost negligible as compared to the contribution due to size quantization (especially for exited states), so that the energy spectrum manifests nearly perfect periodicity as a function of $B$. The axial energy quantization rapidly weakens with increasing height of the nanocylinder. As a result, at $\eta \gg 1$, the relative role of the Zeeman splitting becomes quite pronounced, leading to visible aperiodicity of the patterns of electron energies versus $B$.

Let us consider the impact of the aforedescribed behavior of the single-electron energy spectrum on the Aharonov-Bohm oscillations in the magnetic moment of $N$-electron core-shell nanocylinders with different height-to-radius aspect ratio $\eta$. 

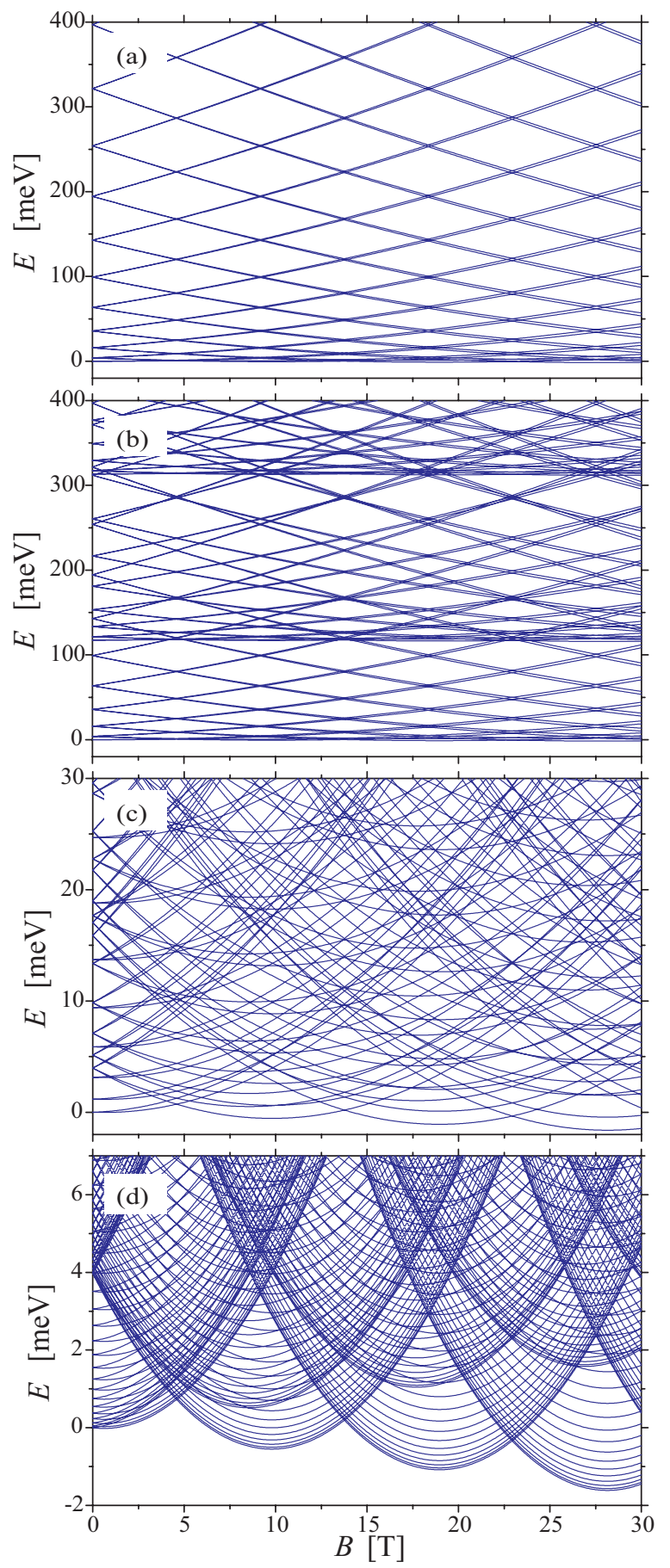

FIG. 2. (Color online) Lowest single-electron energy levels in a cylindrical shell with radius $R=12 \mathrm{~nm}$ and thickness $d \rightarrow 0$, as a function of the applied magnetic field $B(\alpha=0)$ for different height-to-radius ratio: $h / R=0.1$ (a), $h / R=1$ (b), $h / R=10$ (c), and $h / R=50(\mathrm{~d})$.

The magnetic moment of an electron in the state with quantum numbers $L, n$, and $s$,

$$
\mu_{L s}=-\frac{\partial E_{L n s}}{\partial B}=-\mu_{B}\left[\left(L+\frac{\pi R^{2} B}{\Phi_{0}}\right) \frac{m_{0}}{m_{e}}+g s\right],
$$

does not depend on the quantum number $n$, related to the quantization of electron motion along the cylinder axis. The
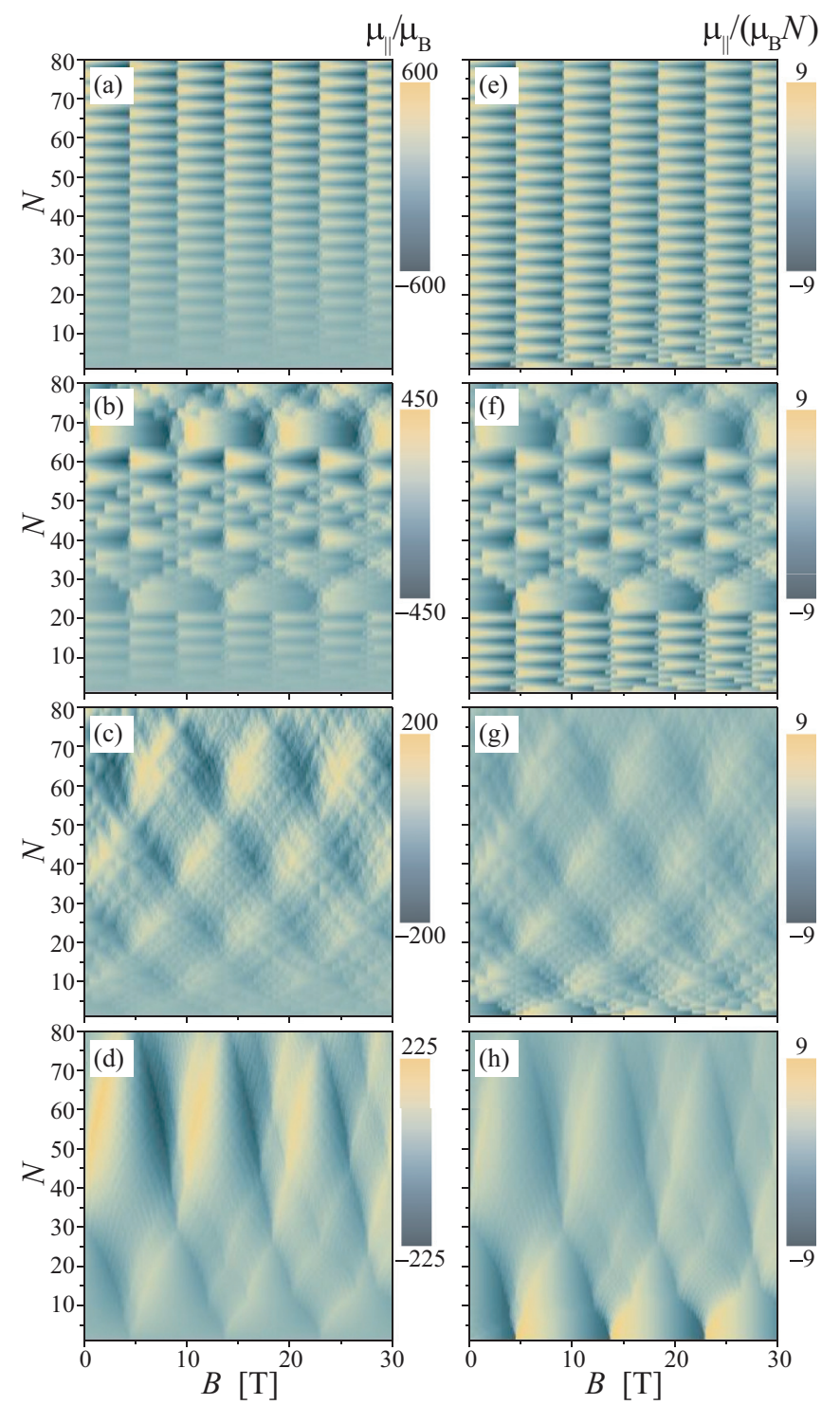

FIG. 3. (Color online) Ground-state magnetic moment $\mu$ of $N$ noninteracting electrons [(a) to (d)] and magnetic moment per electron [(e) to (h)] as a function of the applied magnetic field $B$ ( $\alpha=0$ ) for thin-wall hollow cylinders with radius $R=12 \mathrm{~nm}$ and different height-to-radius ratios: $h / R=0.1$ [(a) and (e)], $h / R=1$ [(b) and (f)], $h / R=10[(\mathrm{c})$ and (g) $]$, and $h / R=50$ [(d) and (h)].

total magnetic moment of electrons in the cylindrical shell can be written as

$$
\mu=\sum_{L, n, s} f_{L n s} \mu_{L s}
$$

where $f_{\text {Lns }}$ is the occupation probability for the state, characterized by quantum numbers $L, n$, and $s$. In Fig. 3, the magnetic moment $\mu$, calculated from Eq. (8) for cylinders with different height-to-radius ratio $\eta$ and different number $N$ of noninteracting electrons at zero temperature, is plotted as a function of the magnetic field $B$, parallel to the $z$ axis.

In the case $\eta \ll 1$ [see Figs. 3(a) and 3(e)], the patterns of $\mu(B, N)$ almost exactly reproduce the results ${ }^{30}$ for a $1 \mathrm{D}$ ring with noninteracting electrons (small differences, observable mainly for relatively small $N$ at relatively high magnetic fields, 
are caused by the Zeeman effect in the present calculations). At $N \gg 1$, the magnetic moment becomes a nearly periodic function of the number of electrons $N$ with period $\Delta N=4$. For weak fields $B$, the response of a multielectron ring is diamagnetic in the case where the number of electrons is even while the number of "electron pairs" is odd $(N \bmod 4=2)$, otherwise the current is paramagnetic (the case of a singleelectron ring is an exception). As seen from Fig. 3(e), for a given value of $N \bmod 4$, the amplitude of the AB oscillations of $\mu(B)$ for an $N$-electron structure increases with $N$ linearly. Only in the case of an even number of electrons or $N=1$, the magnetic moment $\mu(B, N)$ exhibits, as a function of $B$, oscillations with period $\Phi_{0} /\left(\pi R^{2}\right)$, typical for a singleelectron 1D quantum ring with radius $R$. For a large odd number $N$, this period becomes two times smaller, $\Phi_{0} /\left(2 \pi R^{2}\right)$. Also, for an ensemble of $1 \mathrm{D}$ rings with a large number of noninteracting electrons per ring and a significant dispersion of this number within the ensemble, the period of the AB oscillations versus magnetic field appears to be $\Phi_{0} /\left(2 \pi R^{2}\right) .^{30}$

In the case of Figs. 3(a) and 3(e) $(\eta=0.1)$, all the electrons under consideration are in the lowest state of the " $z$ quantization" $(n=1)$. When increasing the height of the cylinder to $h=R$, also states with $n>1$ become populated for $N>20$. Therefore, the aforedescribed behavior of $\mu(B, N)$ is manifested only for $N \leqslant 20$, while at larger values of $N$ the patterns of $\mu(B, N)$ are more complicated [see Figs. 3(b) and 3(f)]. At $\eta \gg 1$, due to a relative increase of the Zeeman-interaction contribution to the electron energy, crossings between energy levels corresponding to magnetic quantum numbers $L$ and $L-1$ occur at different magnetic fields for different electron spins and this difference is larger at higher fields $B$ [see Fig. 2(d)]. As a result, the extrema of $\mu(B)$, which correspond to the crossing fields, tend to split and the $\mathrm{AB}$ oscillations of $\mu(B)$ are not perfectly periodic. Both effects are more pronounced at $N \gg 1$ [see Figs. 3(d) and $3(\mathrm{~h})]$. An additional fine structure of the $\mu(B)$ patterns shown in Figs. 3(c), 3(d), 3(g), and 3(h) arises from the dependence of the aforementioned crossing fields on the " $z$-quantization" number $n$, which is different for different electrons [see Figs. 2(c) and 2(d)]. Nevertheless, the shape of the calculated magnetization oscillations remains much more regular as compared to the case of multielectron quantum dots, ${ }^{31-33}$ where the magnetic field strongly affects the electron energy quantization in the radial direction.

From Figs. 3(f) to 3(h), one can further deduce that for sufficiently large $N$ (for $N>20$ in the case of $h=R$ ), the AB oscillation amplitude per electron decreases with $N$. This trend is somewhat more pronounced for $h \gg R$. Nevertheless, even for $\eta \gg 1$, the amplitude of the AB oscillations of $\mu(B, N)$ in core-shell nanocylinders with $N \gg 1$ remains sufficiently large, although it is reduced as compared to a 1D nanoring with the same $N$ [compare Figs. 3(c) and 3(d) to 3(a)]. In this connection, it is worth mentioning that for realistic nanostructures with finite potential barriers and repulsive electron-electron interaction, confinement of $N \gg 1$ electrons in a quasi-1D quantum ring, similar to that studied in Refs. 18, 19, and 25 is more difficult to achieve as compared to the case of a cylindrical shell with the same radius and $h / R \gg 1$, where the number of confined single-electron states is much larger than in a quasi-1D quantum ring. Remarkably, for sufficiently large values of the height-to-radius ratio, there exist wide ranges of $N$, for which the period and the phase of the AB oscillations of $\mu(B, N)$ do not depend on $N$ [see, e.g., the results for $1 \leqslant N \leqslant 20$ and $40 \leqslant N \leqslant 80$ in Figs. 3(d) and 3(h)]. As implied by Figs. 3(d) and 3(h), for an ensemble of multielectron core-shell nanocylinders, where $N$ varies within one of the aforementioned $N$ ranges, the $\mathrm{AB}$ oscillations of the magnetic moment would have the same period $\Phi_{0} /\left(\pi R^{2}\right)$ as that of single-electron 1D nanorings, but with a significantly enhanced amplitude.

Since the above results and conclusions correspond to a greatly oversimplified model, it is worth examining how sensitive they are to different factors present in realistic coreshell cylindrical nanostructures. In this section, we address the effect of two such factors: a nonzero thickness of the shell and a possible misalignment of the applied magnetic field with respect to the cylinder axis.

\section{B. Cylindrical shell with nonzero thickness}

In the case of a hard-wall cylindrical potential well with a nonvanishing thickness, the electron wave function can be written as

$$
\Psi_{L n k}(\rho, \varphi, z)=\frac{1}{\sqrt{2 \pi}} e^{i L \varphi} \chi_{n}(z) \psi_{L k}(\rho),
$$

where $k$ labels the radial solutions for a given $L$, the $z$ functions $\chi_{n}(z)$ are given by Eqs. (4) and (5), and the radial functions $\psi_{L k}$ satisfy the equation

$$
\begin{aligned}
& \frac{\hbar^{2}}{2 m_{e}}\left[\frac{\partial^{2} \psi_{L k}}{\partial \rho^{2}}+\frac{1}{\rho} \frac{\partial \psi_{L k}}{\partial \rho}-\frac{L^{2}}{\rho^{2}} \psi_{L k}-\left(\frac{e B}{2 \hbar}\right)^{2} \rho^{2} \psi_{L k}\right] \\
& \quad+\epsilon_{L k} \psi_{L k}=0 .
\end{aligned}
$$

The eigenvalue $\epsilon_{L k}$ is linked to the total single-electron energy $E_{\text {Lnks }}$ by the expression

$$
E_{L n k s}=\frac{\hbar^{2}}{2 m_{e} R^{2}}\left(\frac{\pi n}{\eta}\right)^{2}+\epsilon_{L k}+\mu_{B} \frac{m_{0}}{m_{e}} L B+\mu_{B} g s B .
$$

Instead of following a cumbersome procedure expressing the general solution of Eq. (10) in terms of confluent hypergeometric functions and applying the relevant boundary conditions, we have numerically solved Eq. (10), expanding the wave functions $\psi_{L k}$ within a finite basis of standing radial waves, which already satisfy the necessary boundary conditions. The calculations of single-electron energy levels, corresponding to the lowest state of radial quantization, have been performed for a cylindrical shell with mean radius $R=12 \mathrm{~nm}$, heightto-radius ratio $\eta=50$, and thickness $d=5 \mathrm{~nm}$. The resulting patterns of $\mu(B, N)$ and $\mu(B, N) / N$ (not shown here) appear almost undistinguishable from those displayed in Figs. 3(d) and $3(\mathrm{~h})$, indicating that the particular value of the shell thickness $(d \ll R)$ has a rather weak effect on the magnetic moment $\mu(B, N)$ of core-shell nanocylinders with $\eta \gg 1$. For this reason, we restrict ourselves to the case of $d \rightarrow 0$.

\section{Cylindrical shell in a tilted magnetic field}

Let us now consider the experimentally relevant case of a nonzero angle $\alpha$ between the field $\mathbf{B}$ and the cylinder axis. This 
case can be relevant, e.g., for an ensemble of nanocylinders with slightly misaligned axes or when measuring the magnetic moment $\mu(B)$ of nanostructures with a torque magnetometer. ${ }^{18}$ Assuming that the applied field $\mathbf{B}$ lies in the $x z$ plane and choosing the gauge $A_{\rho}=0, A_{\varphi}=B \rho \cos (\alpha) / 2, A_{z}=$ $B \rho \sin (\alpha) \sin (\varphi)$ for the vector potential, the single-electron Schrödinger equation takes the form

$$
\begin{aligned}
& \frac{\hbar^{2}}{2 m_{e}}\left(-\frac{i}{R} \frac{\partial}{\partial \varphi}+\frac{e B R \cos (\alpha)}{2 \hbar}\right)^{2} \Psi \\
& \quad+\frac{\hbar^{2}}{2 m_{e}}\left(-i \frac{\partial}{\partial z}+\frac{e B R \sin (\alpha)}{\hbar} \sin (\varphi)\right)^{2} \Psi+\mu_{B} g s B \Psi \\
& =E \Psi .
\end{aligned}
$$

For $\alpha \neq 0$, the second term in the left-hand side of Eq. (12) depends on both $z$ and $\varphi$, so that a simple scheme with separation of variables is not applicable. We solve the Schrödinger equation (12) numerically by diagonalizing the matrix Hamiltonian in a finite basis, formed by the 300 lowest single-electron states in a magnetic field parallel to the $z$ axis and equal to $B \cos (\alpha)$. The corresponding matrix elements of the Hamiltonian are

$$
H_{L n s, L^{\prime} n^{\prime} s}=E_{L n s} \delta_{L L^{\prime}} \delta_{n n^{\prime}}+V_{L n, L^{\prime} n^{\prime}}+W_{L n, L^{\prime} n^{\prime}},
$$

where the energy $E_{L n s}$ of a basis state is

$$
E_{L n s}=\frac{\hbar^{2}}{2 m_{e}}\left(\frac{\pi n}{h}\right)^{2}+\left(\frac{L}{R}+\frac{e B \cos (\alpha)}{2 \hbar}\right)^{2}+\mu_{B} g s B .
$$

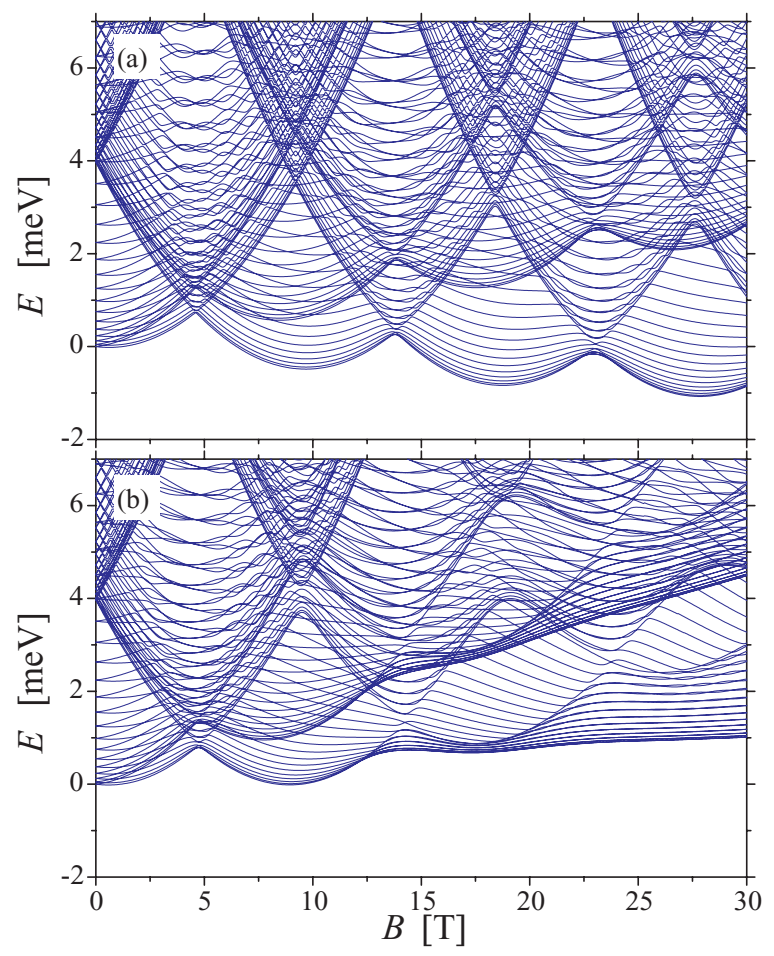

FIG. 4. (Color online) Lowest single-electron energy levels in a thin-wall hollow cylinder with radius $R=12 \mathrm{~nm}$ and height-to-radius ratio $h / R=50$ as a function of the applied magnetic field $B$ for different angles between the field and the cylinder axis: $\alpha=5^{\circ}$ (a) and $\alpha=15^{\circ}(\mathrm{b})$.
The terms

$$
\begin{aligned}
V_{L n, L^{\prime} n^{\prime}}= & \frac{e^{2} R^{2} B^{2} \sin ^{2}(\alpha)}{4 m_{e}} \delta_{n, n^{\prime}} \\
& \times\left(\delta_{L, L^{\prime}}-\frac{1}{2} \delta_{L, L^{\prime}+2}-\frac{1}{2} \delta_{L, L^{\prime}-2}\right)
\end{aligned}
$$

are responsible for the mixing of basis states with the same parity of $L$ and $L^{\prime}$, while the terms

$$
\begin{aligned}
W_{L n, L^{\prime} n^{\prime}}= & \frac{2 e \hbar R B \sin (\alpha)}{m_{e} h}\left(\delta_{L, L^{\prime}-1}-\delta_{L, L^{\prime}+1}\right)(-1)^{\left(n-n^{\prime}-1\right) / 2} \\
& \times\left(\left|n-n^{\prime}\right| \bmod 2\right) \frac{n n^{\prime}}{n^{2}-n^{\prime 2}}
\end{aligned}
$$

mix the states with different parity of $L$ and $L^{\prime}$ and, simultaneously, different parity of $n$ and $n^{\prime}$.

In Fig. 4, the calculated lowest single-electron energy levels in a core-shell nanocylinder with $R=12 \mathrm{~nm}$ and $\eta=50$ are shown for two different values of the angle $\alpha$ between the applied magnetic field and the cylinder axis. As seen from a comparison of Fig. 4 to Fig. 2(d), an increase of $\alpha$ leads to the appearance of numerous anticrossings in the energy spectrum (except for states with different electron spin projections). A misalignment of the field $B$ with respect to the cylinder axis also tends to smooth out the Aharonov-Bohm oscillations of the electron energy levels, especially at strong magnetic fields. In Fig. 5, we plot the ground-state magnetic moment, calculated for cylindrical shells with different $N$, as a function of the applied magnetic field, tilted by $\alpha=5^{\circ}$ and $15^{\circ}$. In line with the aforedescribed results for the energy spectrum, an increase of $\alpha$ is seen to suppress the
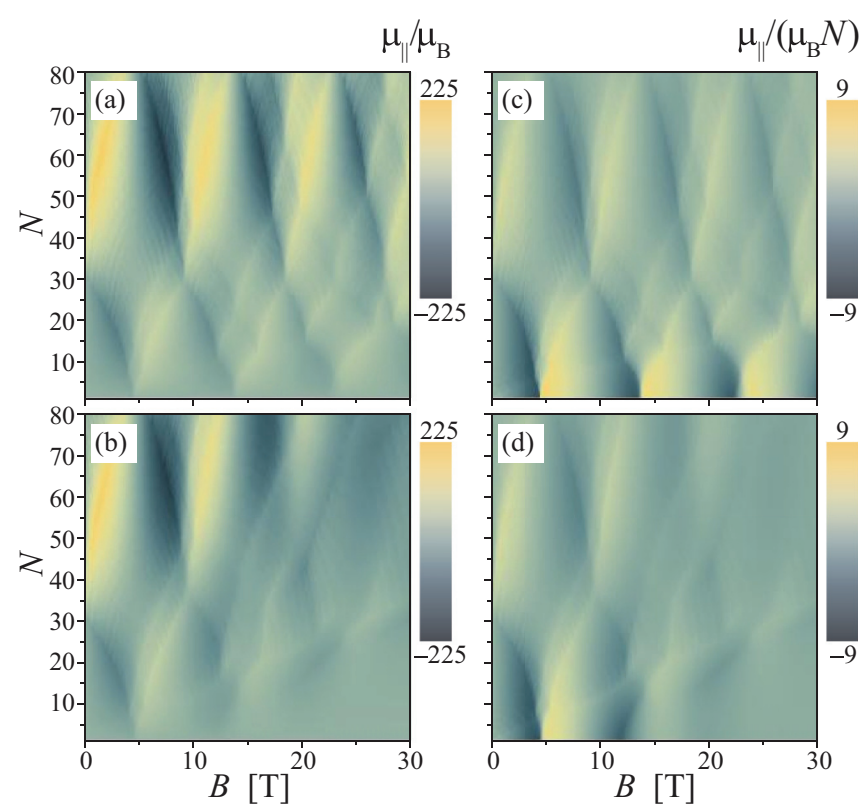

FIG. 5. (Color online) Parallel (to the applied field) components of the ground-state magnetic moment of $N$ noninteracting electrons [(a) and (b)] and of the magnetic moment per electron [(c) and (d)] in a thin-wall hollow cylinder with radius $R=12 \mathrm{~nm}$ and height-to-radius ratio $h / R=50$ as a function of the applied magnetic field $B$ for different angles between the field and the cylinder axis: $\alpha=5^{\circ}[$ (a) and (c)] and $\alpha=15^{\circ}[(\mathrm{b})$ and (d)]. 
Aharonov-Bohm oscillations of the magnetic moment $\mu(B)$ at high magnetic fields. At the same time, the first oscillation remains almost unaffected even for a rather strong field misalignment corresponding to $\alpha=15^{\circ}$. For smaller angles $\alpha$, the effect of this misalignment on the $\mathrm{AB}$ oscillations can hardly be considered as dramatic, although it is not fully negligible.

\section{EFFECTS OF THE ELECTRON-ELECTRON AND ELECTRON-IMPURITY INTERACTIONS ON THE AHARONOV-BOHM OSCILLATIONS}

\section{A. Formalism}

Here, the ground-state energy and the corresponding magnetic moment of an $N$-electron core-shell nanocylinder are analyzed taking into account the interaction of the electrons in the shell with each other as well as with positively charged impurities located in the core. The number of these impurities is assumed to coincide with the number of conduction electrons, so that the nanocylinder as a whole is electrically neutral.

We consider an $N$-electron system, described by the Schrödinger equation

$$
\left[\sum_{i=1, N}^{N} H_{i}^{(1)}+\sum_{i=1, N}^{N} \sum_{j=i+1}^{N} V_{e e}\left(\mathbf{r}_{i}-\mathbf{r}_{j}\right)\right] \Psi^{(N)}=E^{(N)} \Psi^{(N)},
$$

where the first term in square brackets is a sum of "singleparticle" Hamiltonians of noninteracting electrons and the second term describes the electron-electron Coulomb interaction. For simplicity, in the present calculations we assume a uniform dielectric constant $\varepsilon_{r}=10$ for the cylindrical core-shell structure and the surrounding medium (the capping layer) and take $V_{e e}\left(\mathbf{r}_{i}-\mathbf{r}_{j}\right)$ in the form

$$
V_{e e}\left(\mathbf{r}_{i}-\mathbf{r}_{j}\right)=\frac{e^{2}}{4 \pi \varepsilon_{0} \varepsilon_{r}\left|\mathbf{r}_{i}-\mathbf{r}_{j}\right|} .
$$

Here, $\varepsilon_{0}$ is the vacuum permittivity. The single-electron Hamiltonian $H_{i}^{(1)}$ is written as

$$
H_{i}^{(1)}=h_{i}^{(1)}+\mu_{B} g s_{i} B
$$

with

$$
h_{i}^{(1)}=\frac{\hbar^{2}}{2 m_{e}}\left(-i \nabla_{i}+\frac{e}{\hbar} \mathbf{A}\right)^{2}+V_{e i}\left(\mathbf{r}_{i}\right) .
$$

The last term in Eq. (20) describes the electron-impurity interaction

$$
V_{e i}\left(\mathbf{r}_{i}\right)=-\frac{e^{2}}{4 \pi \varepsilon_{0} \varepsilon_{r}} \sum_{k=1}^{N} \frac{1}{\left|\mathbf{r}_{i}-\mathbf{R}_{k}\right|},
$$

where $\mathbf{R}_{k}=\left(\rho_{k}, \varphi_{k}, z_{k}\right)$ is the position of the $k$ th impurity.

The $N$-electron wave function $\Psi^{(N)}$ is modeled by a single Slater determinant and calculated within the HartreeFock approximation (see, e.g., Ref. 34). The corresponding Schrödinger equation for the single-particle Hartree-Fock coordinate wave functions of spin-up $s=\frac{1}{2}$ and spin-down $s=-\frac{1}{2}$ electrons can be written in the form

$$
\begin{aligned}
& {\left[h^{(1)}(\mathbf{r})+C(\mathbf{r})\right] \Phi_{s, k}(\mathbf{r})-\int d^{3} r K_{s, k}\left(\mathbf{r}, \mathbf{r}^{\prime}\right) \Phi_{s, k}\left(\mathbf{r}^{\prime}\right)} \\
& \quad=\epsilon_{s, k} \Phi_{s, k}(\mathbf{r}), \quad s=\frac{1}{2},-\frac{1}{2}
\end{aligned}
$$

where

$$
C(\mathbf{r})=\sum_{s= \pm 1 / 2} \sum_{k=1}^{N_{s}} \int d^{3} r^{\prime} V_{e e}\left(\mathbf{r}-\mathbf{r}^{\prime}\right)\left|\Phi_{s, k}\left(\mathbf{r}^{\prime}\right)\right|^{2}
$$

and

$$
K_{s}\left(\mathbf{r}, \mathbf{r}^{\prime}\right)=\sum_{k=1}^{N_{s}} V_{e e}\left(\mathbf{r}-\mathbf{r}^{\prime}\right) \Phi_{s, k}^{*}\left(\mathbf{r}^{\prime}\right) \Phi_{s, k}(\mathbf{r}) .
$$

$N_{s}$ is the number of electrons with spin projection $s\left(N_{-1 / 2}+\right.$ $\left.N_{1 / 2}=N\right)$. The expectation value for the Hamiltonian (17) is expressed through the eigenvalues $\epsilon_{s, k}$ of Eqs. (22) as

$$
\begin{aligned}
E^{(N)}= & \sum_{s= \pm 1 / 2} \sum_{k=1}^{N_{s}}\left[\epsilon_{s, k}-\frac{1}{2}\left(C_{s, k}-E_{s, k}\right)\right] \\
& +\frac{1}{2}\left(N_{1 / 2}-N_{-1 / 2}\right) \mu_{B} g B,
\end{aligned}
$$

where the direct and exchange energy contributions are determined by

$$
C_{s, k}=\int d^{3} r C(\mathbf{r})\left|\Phi_{s, k}(\mathbf{r})\right|^{2}
$$

and

$$
E_{s, k}=\int d^{3} r \int d^{3} r^{\prime} K_{s}\left(\mathbf{r}, \mathbf{r}^{\prime}\right) \Phi_{s, k}^{*}(\mathbf{r}) \Phi_{s, k}\left(\mathbf{r}^{\prime}\right),
$$

respectively.

Equations (22) are solved numerically, expanding the functions $\Phi_{s, k}(\mathbf{r})$ over the lowest-state eigenfunctions of the corresponding single-electron problem without the electronimpurity interaction

$$
\Psi_{j}(\mathbf{r}) \equiv \Psi_{L_{j} n_{j}}(\rho, \varphi, z)=\frac{1}{\sqrt{2 \pi}} e^{i L_{j} \varphi} \chi_{n_{j}}(z) \delta(R-\rho),
$$

where $\chi_{n_{j}}(z)$ are given by Eqs. (4) and (5). Note that a particular set of basis functions depends on the applied magnetic field $B$. Using the electron-impurity interaction

$$
\Phi_{s, k}(\mathbf{r})=\sum_{i=1}^{n_{\text {bas }}} a_{s, k, j} \Psi_{j}(\mathbf{r})
$$

the iterative equations for the expansion coefficients $a_{s, k, j}$ at a given number $N_{-1 / 2}$ of spin-down electrons take the form

$$
\begin{aligned}
\sum_{i=1}^{n_{\text {bas }}}\left[\left\langle\Psi_{j}\left|h^{(1)}\right| \Psi_{i}\right\rangle+A_{j i}-B_{s j i}\right] a_{s, k, i}^{(\text {new })} & =\epsilon_{s, k}\left(N_{-1 / 2}\right) a_{s, k, j}^{(\text {new })}, \\
j & =1, n_{\text {bas }}
\end{aligned}
$$

where $n_{\text {bas }}$, taken to be $n_{\text {bas }}=250$ in the present calculations, is the length of the used basis. The "interaction matrices" 
given by

$$
\begin{aligned}
A_{j i}= & \sum_{s^{\prime}= \pm 1 / 2} \sum_{k^{\prime}=1}^{N_{s^{\prime}}} \sum_{i^{\prime}, j^{\prime}=1}^{n_{\text {bas }}} a_{s^{\prime}, k^{\prime}, j^{\prime}}^{(\text {old }) *} a_{s^{\prime}, k, i^{\prime}}^{(\text {old })} \\
& \times\left\langle\Psi_{j}(\mathbf{r}), \Psi_{j^{\prime}}\left(\mathbf{r}^{\prime}\right)\left|V_{e e}\left(\mathbf{r}-\mathbf{r}^{\prime}\right)\right| \Psi_{i}(\mathbf{r}), \Psi_{i^{\prime}}\left(\mathbf{r}^{\prime}\right)\right\rangle \\
\left(j, i=1, n_{\text {bas }}\right) \text { and } & \\
B_{s j i}= & \sum_{k^{\prime}=1}^{N_{s}} \sum_{i^{\prime}, j^{\prime}=1}^{n_{\text {bas }}} a_{s, k^{\prime}, j^{\prime}}^{(\text {old }) *} a_{s, k^{\prime}, i^{\prime}}^{\text {(old })} \\
& \times\left\langle\Psi_{j}(\mathbf{r}), \Psi_{j^{\prime}}\left(\mathbf{r}^{\prime}\right)\left|V_{e e}\left(\mathbf{r}-\mathbf{r}^{\prime}\right)\right| \Psi_{i^{\prime}}(\mathbf{r}), \Psi_{i}\left(\mathbf{r}^{\prime}\right)\right\rangle
\end{aligned}
$$

correspond to the direct and exchange terms, respectively.

The matrix elements of the single-electron Hamiltonian $h^{(1)}$ are

$$
\begin{aligned}
\left\langle\Psi_{j}\left|h^{(1)}\right| \Psi_{i}\right\rangle= & \delta_{j i} \frac{\hbar^{2}}{2 m_{e} R^{2}}\left[\left(\frac{\pi n_{j}}{\eta}\right)^{2}+\left(L_{j}+\frac{e B R^{2}}{2 \hbar}\right)^{2}\right] \\
& +\left\langle\Psi_{j}\left|V_{e i}\right| \Psi_{i}\right\rangle .
\end{aligned}
$$

The matrix elements of the electron-impurity interaction can be expressed as

$$
\left\langle\Psi_{j}\left|V_{e i}\right| \Psi_{i}\right\rangle=-\frac{e^{2}}{4 \pi^{2} \varepsilon_{0} \varepsilon_{r} h} \sum_{k=1}^{N} w_{k}\left(n_{j}, n_{i}, L_{i}-L_{j}\right)
$$

with

$$
\begin{aligned}
w_{k}\left(n, n^{\prime}, L\right)= & \int_{-h / 2}^{h / 2} d z \frac{\chi_{n}(z) \chi_{n}^{\prime}(z)}{\sqrt{R^{2}+\rho_{k}^{2}+\left(z-z_{k}\right)^{2}}} \\
& \times p_{k L}\left(\frac{2 R \rho_{k}}{R^{2}+\rho_{k}^{2}+\left(z-z_{k}\right)^{2}}\right),
\end{aligned}
$$

where the angular integral is

$$
p_{k L}(x)=\int_{0}^{2 \pi} d \varphi \frac{e^{i L \varphi}}{\sqrt{1-x \cos \left(\varphi-\varphi_{k}\right)}} .
$$

The electron-electron interaction matrix can be written as

$$
\begin{aligned}
& \left\langle\Psi_{j}(\mathbf{r}), \Psi_{j^{\prime}}\left(\mathbf{r}^{\prime}\right)\left|V_{e e}\left(\mathbf{r}-\mathbf{r}^{\prime}\right)\right| \Psi_{i}(\mathbf{r}), \Psi_{i^{\prime}}\left(\mathbf{r}^{\prime}\right)\right\rangle \\
& \quad=\delta_{L_{j}+L_{j^{\prime}}, L_{i}+L_{i^{\prime}}} W\left(n_{j}, n_{j^{\prime}}, n_{i}, n_{i^{\prime}},\left|L_{i}-L_{j}\right|\right)
\end{aligned}
$$

with

$$
\begin{aligned}
& W\left(n, n^{\prime}, p, p^{\prime}, L\right) \\
& \quad=v_{c}\left(n-p, n^{\prime}-p^{\prime}, L\right)-s_{(++)} v_{c}\left(n+p, n^{\prime}+p^{\prime}, L\right) \\
& \quad+s_{(-+)} v_{c}\left(n-p, n^{\prime}+p^{\prime}, L\right)+s_{(+-)} v_{c}\left(n+p, n^{\prime}-p^{\prime}, L\right) \\
& \quad \text { for } \beta_{(++)} \bmod 2=0 \text { and }\left(\beta_{n}+\beta_{p}\right) \bmod 2=0, \quad(38)
\end{aligned}
$$

$$
\begin{aligned}
& W\left(n, n^{\prime}, p, p^{\prime}, L\right) \\
& \quad=v_{s}\left(n+p, n^{\prime}+p^{\prime}, L\right)-s_{(--)} v_{s}\left(n-p, n^{\prime}-p^{\prime}, L\right) \\
& \quad-s_{(-+)} v_{s}\left(n-p, n^{\prime}+p^{\prime}, L\right)-s_{(+-)} v_{s}\left(n+p, n^{\prime}-p^{\prime}, L\right) \\
& \quad \text { for } \beta_{(++)} \bmod 2=0 \text { and }\left(\beta_{n}+\beta_{p}\right) \bmod 2=1, \quad(39)
\end{aligned}
$$

and

$$
W\left(n, n^{\prime}, p, p^{\prime}, L\right)=0 \quad \text { for } \quad \beta_{(++)} \bmod 2 \neq 0 .
$$

The integrals, entering the above equations, are given by the following formulas:

$$
\begin{aligned}
v_{c}\left(n, n^{\prime}, L\right)= & \frac{e^{2}}{16 \pi^{3} \varepsilon_{0} \varepsilon_{r} R} \int_{-1 / 2}^{1 / 2} d z \\
& \times \int_{-1 / 2}^{1 / 2} d z^{\prime} \cos (\pi n z) \cos \left(\pi n^{\prime} z^{\prime}\right) Q_{L}\left[\eta\left(z-z^{\prime}\right)\right]
\end{aligned}
$$

and

$$
\begin{aligned}
v_{s}\left(n, n^{\prime}, L\right)= & \frac{e^{2}}{16 \pi^{3} \varepsilon_{0} \varepsilon_{r} R} \int_{-1 / 2}^{1 / 2} d z \\
& \times \int_{-1 / 2}^{1 / 2} d z^{\prime} \sin (\pi n z) \sin \left(\pi n^{\prime} z^{\prime}\right) Q_{L}\left[\eta\left(z-z^{\prime}\right)\right],
\end{aligned}
$$

where the angular integral is

$$
Q_{L}(x)=2 \pi \int_{0}^{2 \pi} d \varphi \frac{\cos L \varphi}{\sqrt{2(1-\cos \varphi)+x^{2}}} .
$$

The parity-related coefficients $\beta_{n}, \beta_{( \pm \pm)}$, and $s_{( \pm \pm)}$are determined as

$$
\beta_{n}=1+n \bmod 2
$$

$$
\begin{gathered}
\beta_{( \pm \pm)}=\left|\left(\beta_{n} \pm \beta_{p}\right)+\left(\beta_{n^{\prime}} \pm \beta_{p^{\prime}}\right)\right|, \\
s_{( \pm \pm)}= \begin{cases}1 & \text { for } \quad \beta_{( \pm \pm)} \bmod 4 \neq 0, \\
-1 & \text { for } \quad \beta_{( \pm \pm)} \bmod 4=0 .\end{cases}
\end{gathered}
$$

Importantly for practical computation, the coefficients $v_{c}\left(n, n^{\prime}, L\right)$ and $v_{s}\left(n, n^{\prime}, L\right)$ do not depend on the number of electrons $N$ and they are trivially scaled with $R$ at a fixed aspect ratio $\eta$.

A well-known weak point of the Hartree-Fock approach is that it does not take into account the electron correlation effects. ${ }^{34}$ However, our calculations (not presented here) of the magnetic moment $\mu(B)$ of two-electron hollow nanocylinders with $\eta \sim 1$ (i.e., for ringlike structures) show that the difference between the Hartree-Fock-based results and the results based on the exact numerical diagonalization of the two-electron Hamiltonian ${ }^{21}$ is less important than the effects due to the direct Coulomb and exchange term. For longer nanocylinders, this difference becomes even less significant and definitely negligible as compared to the dramatic (see below) effect of randomly distributed positively charged impurities on the shape of $\mu(B)$.

\section{B. Magnetic moment of the lowest fully spin-polarized state}

In Fig. 6, the calculated energy $E^{(N)}$ and the corresponding magnetic moment $\mu=-\partial E^{(N)} / \partial B$ are shown as a function of $B$ for the lowest fully spin-polarized $\left(N_{-1 / 2}=N\right)$ state of six electrons in a cylindrical layer with radius $R=12 \mathrm{~nm}$ and height-to-radius ratio $h / R=50$. The results are shown for the cases without interactions, with the electron-electron (ee) interaction only, and with the $e e$ and electron-impurity 

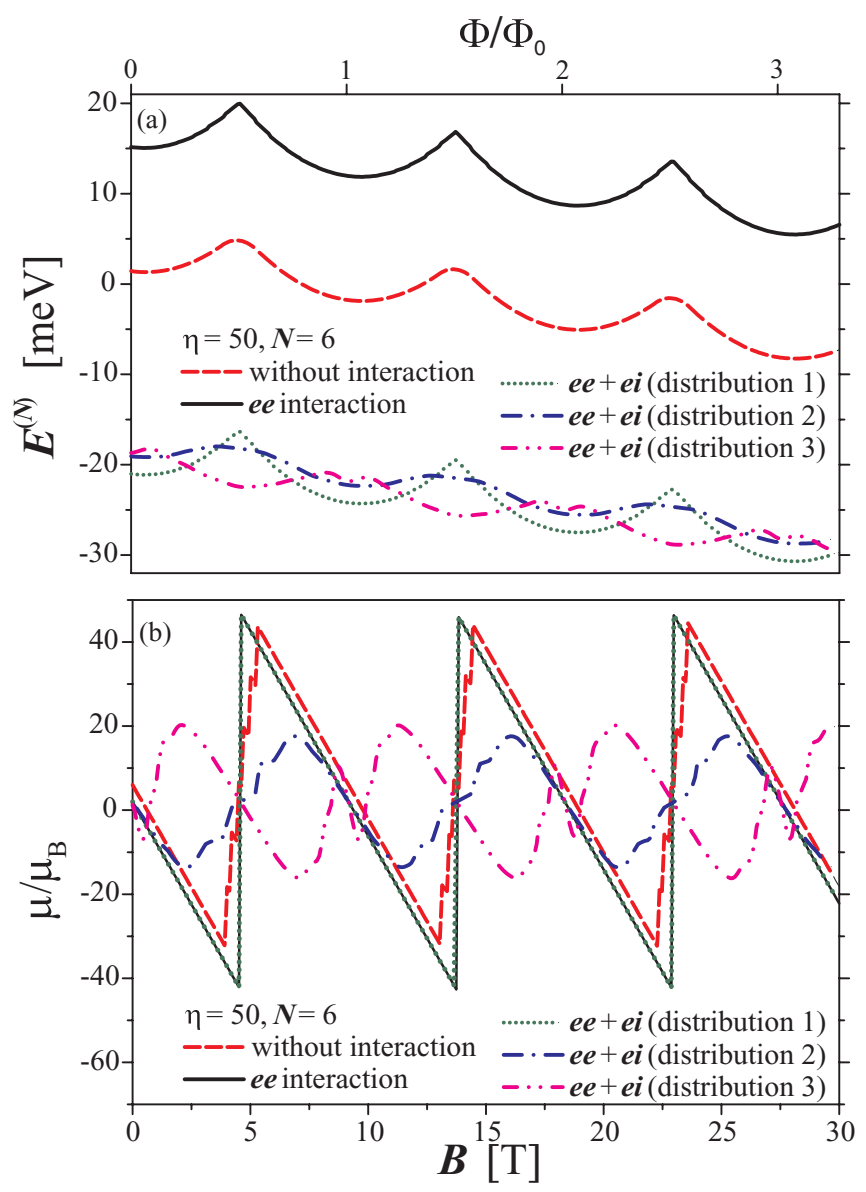

FIG. 6. (Color online) Lowest energy level (a) and the corresponding magnetic moment (b) for $N=6$ fully spin-polarized electrons in a cylindrical layer with radius $R=12 \mathrm{~nm}$ and height-toradius ratio $h / R=50$ as a function of the applied magnetic field $B$ $(\alpha=0)$. The results are shown for the cases without interactions, with the electron-electron (ee) interaction only, and with the ee and electron-impurity (ei) interactions, for three different spatial distributions of the impurities (see Table I in the Appendix).

(ei) interactions for three different spatial distributions of the impurities. The impurity coordinates for the chosen distributions are given in the Appendix. As seen from Fig. 6(a), the main effect of the $e e$ interaction, taken into account alone, is an overall upwards shift of the curve $E^{(N)}(B)$. In addition, the $e e$ interactions tend to make the motion of the electrons "more coherent." As a result, the difference between the magneticfield values, corresponding to crossings of different singleelectron levels with magnetic quantum numbers $L$ and $L-1$ (see the previous section), significantly decreases as compared to the case of noninteracting electrons. Consequently, the "multiple splitting" of minima and maxima of the curves $\mu(B)$, which is typical for noninteracting electrons at $N \gg 1$ and $\eta \gg 1$, can be considerably reduced when taking into account the ee interaction [see Fig. 6(b)].

Figure 6(b) further demonstrates that the incorporation of the electron interaction with positively charged impurities, uniformly distributed along the cylinder axis (distribution 1; see Table I in the Appendix), results only in an overall downwards shift of $E^{(N)}(B)$ without any effect on the shape of the
$\mathrm{AB}$ oscillations of the magnetic moment $\mu(B)$. However, the situation changes dramatically in the case of uncorrelated random impurity distributions, which are much more plausible for realistic nanostructures. ${ }^{35}$ For those distributions (distributions 2 and 3; see Table I in the Appendix), as seen from Fig. 6, the $e i$ interaction significantly modifies the behavior of $E^{(N)}(B)$. This results in an appreciable reduction of the amplitude of the Aharonov-Bohm oscillations for $\mu(B)$. Moreover, the interaction of electrons with randomly distributed impurities strongly affects the shape and the phase of the $\mathrm{AB}$ oscillations. It is obvious that the aforementioned effects can be related, at least to some extent, to the cylindrical-symmetry breaking caused by the presence of randomly distributed impurities. As seen from Table I in the Appendix, some of these impurities are very close to the shell where the electrons are confined, so that the corresponding $e i$ interactions are strong.

At first sight, the aforedescribed undesirable effects of the $e i$ interaction on the $\mathrm{AB}$ oscillations could be significantly reduced by concentrating the impurities in a narrow spatial region close to the cylinder axis, so that the cylindrical symmetry of the system is not strongly affected. Direct measurements of dopant concentrations in nanowires using atom probe tomography ${ }^{36}$ demonstrate that the impurity distribution in the radial direction can be highly inhomogeneous depending on the growth conditions. It may be possible to grow core-shell cylindrical structures similar to those described in Refs. 27-29, with dopants located in a narrow region near the axis. However, as shown below, the effects of the $e i$ interaction on the $\mathrm{AB}$ oscillations can remain quite pronounced even for such nearaxis dopant distributions. In order to illustrate the origin of these effects, we show in Fig. 7 a few examples of the electron probability density distributions along the cylinder axis $P_{e}(z)$. The probability density $P_{e}(z)$ is normalized by the condition $\int_{-h / 2}^{h / 2} P_{e}(z) d z=1$. The calculations are performed for the lowest fully spin-polarized state of $N=4,16$, and 32 electrons in a core-shell nanocylinder with radius $R=15 \mathrm{~nm}$ and heightto-radius ratio $\eta=50$. The impurities are randomly distributed in a region of radius $2.5 \mathrm{~nm}$ near the cylinder axis. The $z$ coordinates of impurities for the chosen distributions are indicated in Fig. 7 with circles. The results are shown for the cases without interactions, with the electron-impurity interaction only, and with both the electron-impurity and electron-electron interactions. Figure 7 clearly shows the electron localization by positively charged impurities. This effect is especially pronounced for impurity (quasi)clusters. Importantly, formation of impurity (quasi)clusters appears rather probable for uncorrelated random impurity distributions. The electron-electron repulsive interaction tends to weaken the localization effect, making the electron probability density distributions along the cylinder axis more uniform. Nevertheless, this distribution remains rather inhomogeneous even in the case when both the electron-impurity and electron-electron interactions are taken into account. As follows from Fig. 7, due to the additional impurity-induced localization, the electron probability density distribution in a core-shell multielectron cylindrical structure can appear similar to that in a stack of few-electron quantum rings or cylinders with a height significantly smaller than $h$.

As illustrated in Figs. 8 to 10, the impurity-induced redistribution of the electron density along the cylinder axis 


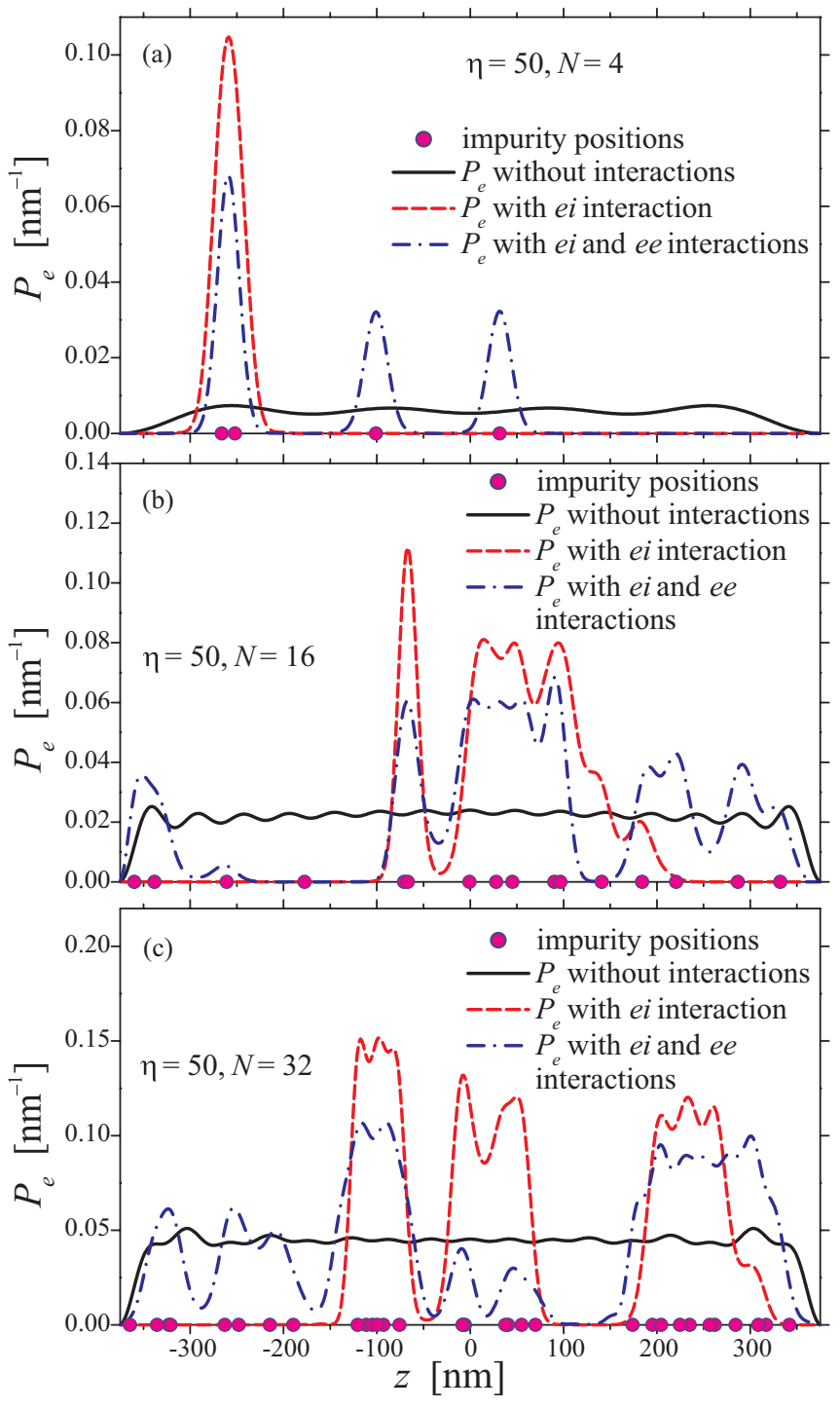

FIG. 7. (Color online) Probability density distribution along the $z$ axis for $N=4$ (a), $N=16$ (b), and $N=32$ (c) fully spin-polarized electrons in a cylindrical layer with radius $R=15 \mathrm{~nm}$ and height-toradius ratio $h / R=50$ at zero applied magnetic field. The results are shown for the cases without interactions, with the electron-impurity (ei) interaction only, and with both the electron-impurity and electronelectron $(e e)$ interactions. The $z$ coordinates of impurities are shown with circles.

has a strong impact on the shape of the $\mathrm{AB}$ oscillations of the magnetic moment $\mu$ versus magnetic field $B$ or the magnetic flux $\Phi$. The shown results correspond to multielectron coreshell nanocylinders with radius $R=15 \mathrm{~nm}$ and height-toradius ratio $\eta=50$. The positively charged impurities are again randomly distributed in a region of radius $2.5 \mathrm{~nm}$ near the cylinder axis. Figure 8(a) illustrates the situation where the amplitude of the $\mathrm{AB}$ oscillations for the fully spin-polarized state of $N=16$ electrons is relatively small in the absence of interactions. The fine oscillations superimposed over the $\mathrm{AB}$ oscillations arise from the fact that, as discussed in Sec. II A, crossings between energy levels corresponding to magnetic quantum numbers $L$ and $L-1$ take place at different magnetic fields for different single-electron energy

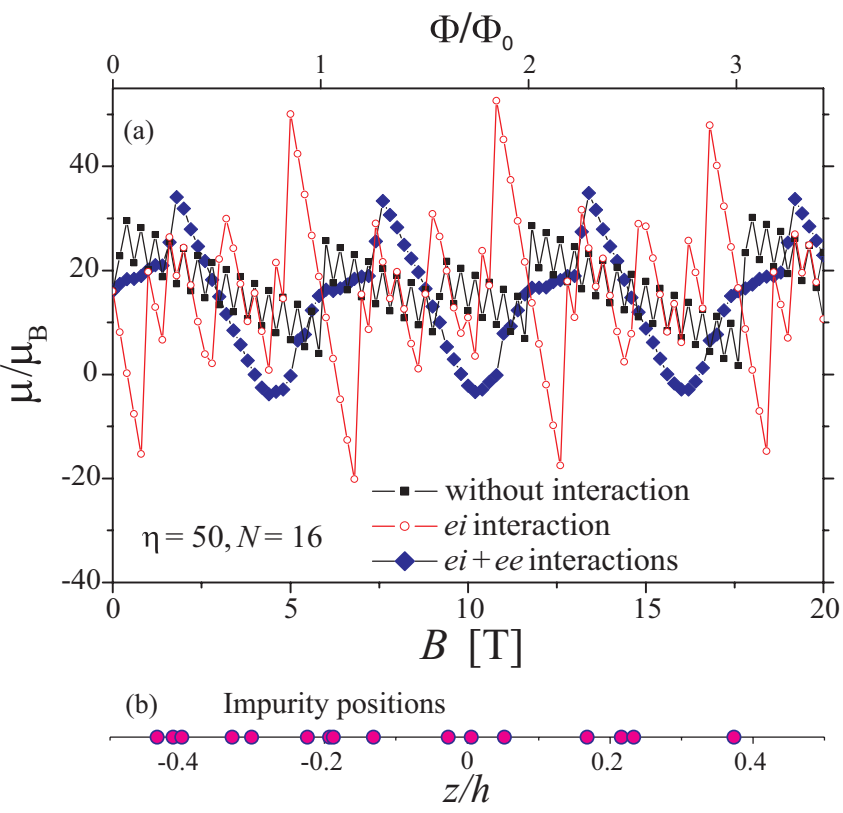

FIG. 8. (Color online) (a) Magnetic moment corresponding to the lowest fully spin-polarized state of $N=16$ electrons in a cylindrical layer with radius $R=15 \mathrm{~nm}$ and height-to-radius ratio $h / R=50$ as a function of the applied homogeneous magnetic field $B(\alpha=0)$. The results are shown for the cases without interactions, with the electron-impurity $(e i)$ interaction only, and with both the electronimpurity and electron-electron (ee) interactions. (b) $z$ coordinates of impurities for the chosen random impurity distribution.

levels. For the impurity distribution shown in Fig. 8(b), the $e i$ interaction, taken alone (without the $e e$ interaction), leads to the appearance of multiple maxima and minima of $\mu(\Phi)$, some of them having a relatively large magnitude. The $e e$ interaction smoothes $\mu(\Phi)$. At the same time, the curve $\mu(\Phi)$, calculated with both the $e i$ and $e e$ interactions taken into account, reveals the appearance of an oscillation component with the period $\Phi_{0} / 2$, expected for a collection of quantum rings, as discussed in the previous section.

\section{Spin polarization and magnetic moment in the ground state}

Another important aspect of the influence of the electronimpurity and electron-electron interactions on the shape of the $\mathrm{AB}$ oscillations relates to the electron spin polarization in the ground state. As seen from Figs. 9(a) and 10(a), in the absence of the $e i$ and $e e$ interactions, the fully spin-polarized ground state for a core-shell nanocylinder with $R=15 \mathrm{~nm}$ and $\eta=50$, containing 12 or 15 electrons, is ultimately established for magnetic fields $B>12.4 \mathrm{~T}$ or $B>18 \mathrm{~T}$, respectively. When taking into account the $e i$ interaction only, the groundstate number of spin-down electrons $N_{-1 / 2}$ fluctuates around values smaller than $N$ within the whole field range under consideration. For this reason, the calculated magnetic moment does not manifest a clear periodicity as a function of the applied magnetic field [see Figs. 9(b) and 10(b)]. In contrast to this, the fully spin-polarized configuration of electrons, interacting both with impurities and with each other, becomes a stable ground state at magnetic fields significantly lower than those in the case of no interactions. This effect is caused by a relatively 

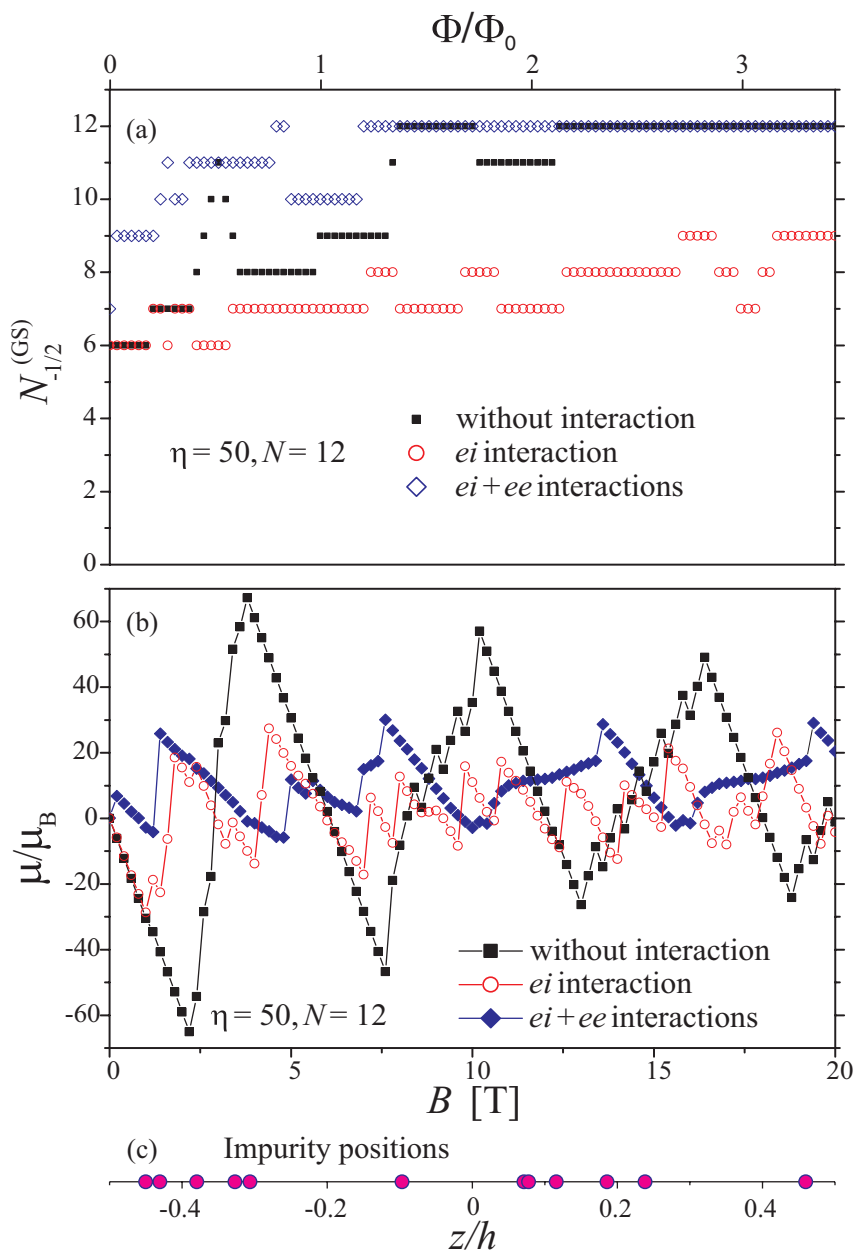

FIG. 9. (Color online) Number of spin-down electrons (a) and magnetic moment (b) in the ground state of $N=12$ electrons in a cylindrical layer with radius $R=15 \mathrm{~nm}$ and height-to-radius ratio $h / R=50$ as a function of the applied magnetic field $B(\alpha=0)$. The results are shown for the cases without interactions, with the electron-impurity $(e i)$ interaction only, and with both the electronimpurity and electron-electron $(e e)$ interactions. The $z$ coordinates of impurities for the selected random impurity distribution are shown in panel (c).

large (negative) exchange energy in the fully spin-polarized state. As a result, the ground-state magnetic moment becomes a periodic function of $B$ already at moderate magnetic fields [see Figs. 9(b) and 10(b)].

As seen from Figs. 8 to 10, although the electron-impurity interaction tends to decrease the amplitude of the AB oscillations for multielectron core-shell nanocylinders, this amplitude remains comparable to that in the case of noninteracting electrons and no impurities. At the same time, the shape and phase of the $\mathrm{AB}$ oscillations for an individual nanocylinder are rather sensitive to a particular impurity distribution [for instance, the curves $\mu(\Phi)$ in Figs. 8 and 9 show the presence of an oscillating component with period $\Phi_{0} / 2$, while in Fig. 10 only oscillations with period $\Phi_{0}$ are present at high magnetic fields]. In connection to this, the question arises as to what happens with the $\mathrm{AB}$ oscillations when the magnetic moment is averaged over an ensemble of core-shell nanocylinders

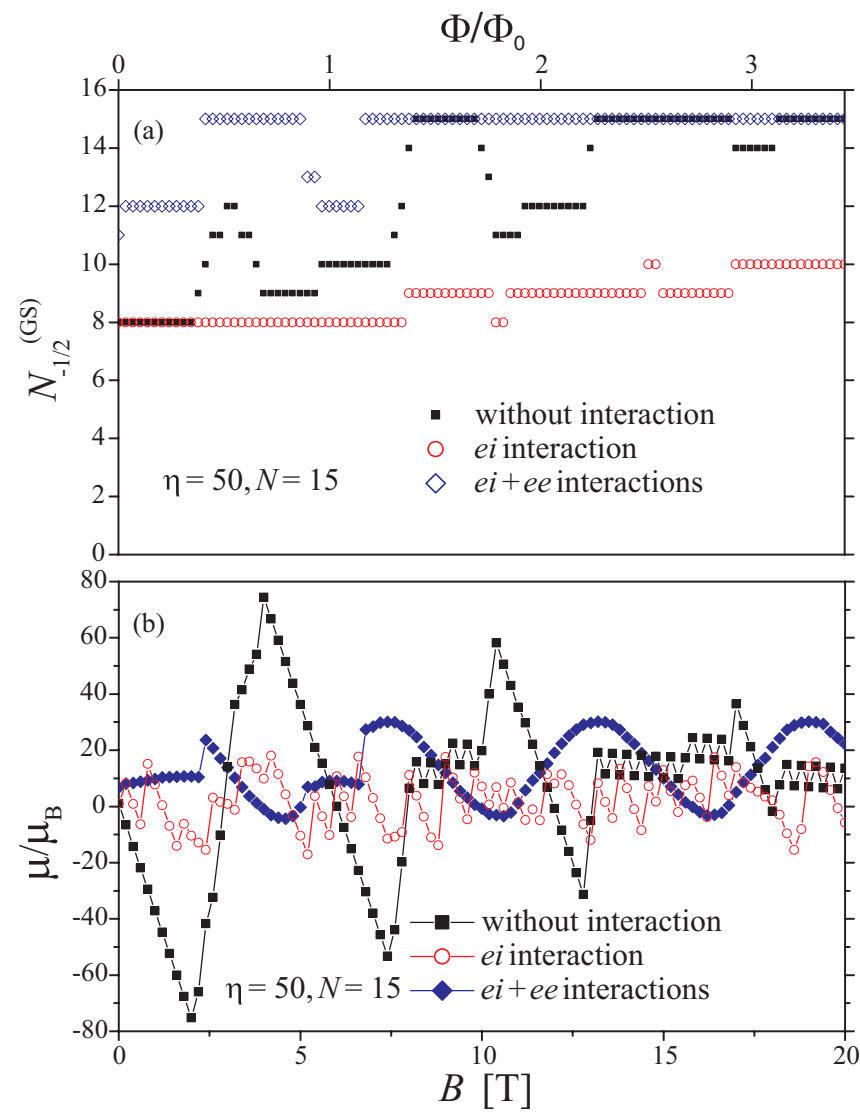

(c) Impurity positions

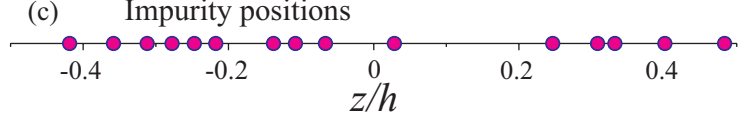

FIG. 10. (Color online) Number of spin-down electrons (a) and magnetic moment (b) in the ground state of $N=15$ electrons in a cylindrical layer with radius $R=15 \mathrm{~nm}$ and height-to-radius ratio $h / R=50$ as a function of the applied magnetic field $B(\alpha=0)$. The results are shown for the cases without interactions, with the electron-impurity $(e i)$ interaction only, and with both the electronimpurity and electron-electron (ee) interactions. The $z$ coordinates of impurities for the selected random impurity distribution are shown in panel (c).

with different impurity distributions. Based on the analysis performed above, one can suggest that the behavior of $\mu(B)$ for such an ensemble should be qualitatively similar to that described in Sec. II for an ensemble of multielectron quantum rings. In general, when both the $e i$ and $e e$ interactions are present, the corresponding numerical simulations for a large assembly of nanocylinders would be extremely time consuming. However, in order to examine qualitatively the above guess, we can limit ourselves to a simpler case where only the $e i$ interaction is taken into account. Indeed, from our previous calculations, we have concluded that the ee interaction has no detrimental effect on the $\mathrm{AB}$ oscillations of the magnetic moment. Moreover, the obtained results confirm that, similarly to the cases of multielectron quantum dots ${ }^{37}$ and 1D systems (see, e.g., Ref. 38), the $e e$ interaction in core-shell nanocylinders significantly weakens the effect of a rough and noisy impurity potential. This means that omitting 


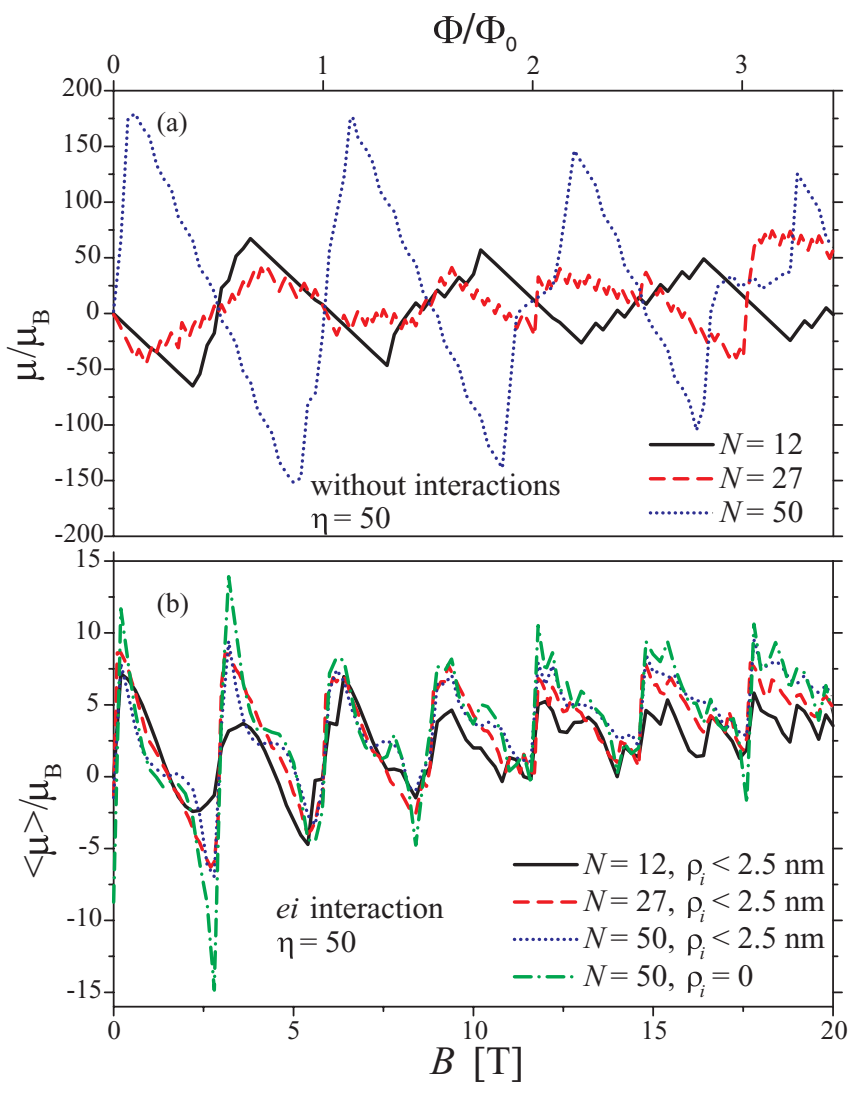

FIG. 11. (Color online) (a) Magnetic moment in the ground state of $N$ noninteracting electrons in a cylindrical layer with radius $R=$ $15 \mathrm{~nm}$ and height-to-radius ratio $h / R=50$ as a function of the applied magnetic field $B(\alpha=0)$ for three different $N$. (b) Averaged magnetic moment in the ground state of $N$ electrons interacting with charged impurities in cylindrical layers with radius $R=15 \mathrm{~nm}$ and heightto-radius ratio $h / R=50$ as a function of the applied magnetic field $B(\alpha=0)$. Each curve corresponds to averaging over 5000 different random distributions of impurities. In the chosen distributions, the impurities are located either near the cylinder axis at distances $\rho_{i}<$ $2.5 \mathrm{~nm}$ from the axis or exactly on the axis $\left(\rho_{i}=0\right)$.

the $e e$ interaction and taking into account only the electron interaction with randomly distributed impurities, one obtains the "most pessimistic prognosis" for the $\mathrm{AB}$ oscillations.

\section{Magnetic moment of an assembly of core-shell nanocylinders}

In Fig. 11, we compare the magnetic moment in the groundstate core-shell nanocylinders with $N$ noninteracting electrons to the magnetic moment $\langle\mu\rangle$ averaged over an assembly of nanocylinders including the $e i$ interaction and 5000 different random impurity distributions. The results of our simulations show that averaging over $\sim 1000$ to 2000 impurity distributions already provides a reasonably good approximation for the amplitude of the $\mathrm{AB}$ oscillations and for the general behavior of $\langle\mu(B)\rangle$ for larger ensembles. As seen from Fig. 11(b), the overall shape of $\langle\mu\rangle$ is qualitatively similar for different $N$, demonstrating $\mathrm{AB}$ oscillations with a twice smaller period than those seen in Fig. 11(a), just in line with our guess, i.e., with what is expected for a collection of rings with different numbers of electrons $<N$. As compared to the case without interactions, the amplitude of the oscillations of $\langle\mu(B)\rangle$ is considerably lower and its dependence on $N$ is rather weak.

For $N=50$, the behavior of $\langle\mu(B)\rangle$ is shown in Fig. 11(b) for the two cases where the impurities are located either near the cylinder axis at distances $\rho_{i}<2.5 \mathrm{~nm}$ from the axis or exactly on the axis $\left(\rho_{i}=0\right)$. As seen Fig. 11(b), random deviations of impurity positions from the cylinder axis, which destroy the cylindrical symmetry of the system, lead to a decrease of the $\mathrm{AB}$ oscillation amplitude. At the same time, for the relatively small deviations under consideration $\left(\rho_{i}<2.5 \mathrm{~nm}\right)$, this effect is not really dramatic, so that the $\mathrm{AB}$ oscillations survive. As argued above, the electron-electron interaction is expected to make these oscillations even more pronounced.

\section{CONCLUSIONS}

We have analyzed the energy spectrum and the magnetic moment of multielectron semiconductor core-shell nanocylinders, subjected to a magnetic field, for different height-toradius ratios $\eta$ and numbers of electrons. It is shown that for noninteracting electrons, the period and phase of the Aharonov-Bohm oscillations of the magnetic moment $\mu(B)$ at $\eta \gg 1$ appear independent of $N$ within rather wide $N$ ranges, in contrast to ideal $1 \mathrm{D}$ rings, where the shape of $\mu(B)$ dramatically depends on $N \bmod 4$. Importantly, the amplitude of the AB oscillations in core-shell nanocylinders with $N \gg 1$ can be much larger than that in single-electron ringlike nanostructures. The $\mathrm{AB}$ oscillation amplitude is robust against the experimentally relevant effects of a nonzero shell thickness as well as of a moderate misalignment of the magnetic field with respect to the cylinder axis.

The behavior of $\mu(B)$ is strongly influenced by the interaction of electrons with randomly distributed charged impurities, even in the case when these impurities are located closely to the cylinder axis, so that the cylindrical symmetry is not strongly violated. Due to the formation of impurity

TABLE I. Cylindrical coordinates of impurities for distributions 1 to 3 .

\begin{tabular}{lcccc}
\hline \hline Distrib. No. & $k$ & $\rho / R$ & $\varphi(\mathrm{rad})$ & \multicolumn{1}{c}{$z / h$} \\
\hline 1 & 1 & 0 & 0 & -0.4167 \\
& 2 & 0 & 0 & -0.25 \\
& 3 & 0 & 0 & -0.0833 \\
& 4 & 0 & 0 & 0.0833 \\
& 5 & 0 & 0 & 0.25 \\
& 6 & 0 & 0 & 0.4167 \\
& 1 & 0.6718 & 5.7684 & 0.2026 \\
& 2 & 0.4265 & 5.6303 & 0.4852 \\
& 3 & 0.2727 & 2.2601 & 0.4085 \\
& 4 & 0.5179 & 2.3789 & 0.1255 \\
& 5 & 0.6503 & 5.4317 & 0.0521 \\
& 6 & 0.0514 & 3.25 & -0.1404 \\
& 1 & 0.7169 & 0.8072 & -0.4817 \\
& 2 & 0.8992 & 0.3931 & -0.2054 \\
& 3 & 0.4379 & 2.8732 & -0.4347 \\
& 4 & 0.2009 & 5.3151 & -0.0934 \\
& 5 & 0.9214 & 0.9381 & -0.0501 \\
& 6 & 0.0451 & 1.3115 & -0.1845 \\
\hline \hline
\end{tabular}


(quasi)clusters, the electron distribution in a cylindrical shell with $\eta \gg 1$ becomes similar to that in a stack of few-electron quantum rings. Such an "additional confinement" for electrons strongly affects both the orbital magnetic moment and the spin configuration in the ground state of the multielectron system. The electron-electron interaction weakens these effects and tends to bring the overall shape of $\mu(B)$ closer to that calculated in the absence of interactions. Although the shape and phase of the $\mathrm{AB}$ oscillations in an individual core-shell nanocylinder are sensitive to a particular distribution of charged impurities, $\mathrm{AB}$ oscillations with a sufficiently high amplitude are shown to survive in the magnetic moment averaged over an assembly of nanocylinders with different impurity distributions.

\section{ACKNOWLEDGMENT}

This work was supported by FOM E0610M and NanoNextNl 6B-3A (The Netherlands), the Scientific Research Community Project No. WO.033.09N (Belgium), and by the Fund for Scientific Research-Flanders, Project No. G.0119.12N.

\section{APPENDIX}

In Table I, we indicate the impurity coordinates for the distributions 1 to 3 , which correspond to the results shown in Fig. 6.
${ }^{1}$ Y. Aharonov and D. Bohm, Phys. Rev. 115, 485 (1959).

${ }^{2}$ N. Byers and C. Yang, Phys. Rev. Lett. 7, 46 (1961).

${ }^{3}$ F. Bloch, Phys. Rev. B 2, 109 (1970).

${ }^{4}$ M. Büttiker, Y. Imry, and R. Landauer, Phys. Lett. A 96, 365 (1983).

${ }^{5}$ C. P. Umbach, S. Washburn, R. B. Laibowitz, and R. A. Webb, Phys. Rev. B 30, 4048 (1984).

${ }^{6}$ R. A. Webb, S. Washburn, C. P. Umbach, and R. B. Laibowitz, Phys. Rev. Lett. 54, 2696 (1985).

${ }^{7}$ S. Washburn and R. A. Webb, Rep. Prog. Phys. 55, 1311 (1992).

${ }^{8}$ D. Yu. Sharvin and Yu. V. Sharvin, Pis'ma Zh. Eksp. Teor. Fiz. 34, 285 (1981) [JETP Lett. 34, 272 (1981)].

${ }^{9}$ L. P. Lévy, G. Dolan, J. Dunsmuir, and H. Bouchiat, Phys. Rev. Lett. 64, 2074 (1990).

${ }^{10}$ V. Chandrasekhar, R. A. Webb, M. J. Brady, M. B. Ketchen, W. J. Gallagher, and A. Kleinsasser, Phys. Rev. Lett. 67, 3578 (1991).

${ }^{11}$ D. Mailly, C. Chapelier, and A. Benoit, Phys. Rev. Lett. 70, 2020 (1993).

${ }^{12}$ A. Lorke, R. J. Luyken, A. O. Govorov, J. P. Kotthaus, J. M. García, and P. M. Petroff, Phys. Rev. Lett. 84, 2223 (2000).

${ }^{13}$ R. J. Warburton, C. Schäflein, D. Haft, F. Bickel, A. Lorke, K. Karrai, J. M. García, W. Schoenfeld, and P. M. Petroff, Nature (London) 405, 926 (2000).

${ }^{14}$ R. J. Warburton, C. Schulhauser, D. Haft, C. Schäflein, K. Karrai, J. M. García, W. Schoenfeld, and P. M. Petroff, Phys. Rev. B 65, 113303 (2002).

${ }^{15}$ J. A. Barker, R. J. Warburton, and E. P. O’Reilly, Phys. Rev. B 69, 035327 (2004).

${ }^{16}$ R. Timm, H. Eisele, A. Lenz, L. Ivanova, G. Balakrishnan, D. L. Huffaker, and M. Dahne, Phys. Rev. Lett. 101, 256101 (2008).

${ }^{17}$ C. Heyn, A. Stemmann, C. Strelow, T. Koppen, D. Sonnenberg, A. Graf, S. Mendach, and W. Hansen, J. Nanoelectron. Optoelectron. 6, 62 (2011).

${ }^{18}$ N. A. J. M. Kleemans, I. M. A. Bominaar-Silkens, V. M. Fomin, V. N. Gladilin, D. Granados, A. G. Taboada, J. M. García, P. Offermans, U. Zeitler, P. C. M. Christianen, J. C. Maan, J. T. Devreese, and P. M. Koenraad, Phys. Rev. Lett. 99, 146808 (2007).

${ }^{19}$ V. M. Fomin, V. N. Gladilin, S. N. Klimin, J. T. Devreese, N. A. J. M. Kleemans, and P. M. Koenraad, Phys. Rev. B 76, 235320 (2007).

${ }^{20}$ A. Bruno-Alfonso and A. Latge, Phys. Rev. B 77, 205303 (2008).
${ }^{21}$ V. M. Fomin, V. N. Gladilin, J. T. Devreese, N. A. J. M. Kleemans, and P. M. Koenraad, Phys. Rev. B 77, 205326 (2008).

${ }^{22}$ N. A. J. M. Kleemans, J. H. Blokland, A. G. Taboada, H. C. M. van Genuchten, M. Bozkurt, V. M. Fomin, V. N. Gladilin, D. Granados, J. M. García, P. C. M. Christianen, J. C. Maan, J. T. Devreese, and P. M. Koenraad, Phys. Rev. B 80, 155318 (2009).

${ }^{23}$ F. Ding, N. Akopian, B. Li, U. Perinetti, A. Govorov, F. M. Peeters, C. C. Bof Bufon, C. Deneke, Y. H. Chen, A. Rastelli, O. G. Schmidt, and V. Zwiller, Phys. Rev. B 82, 075309 (2010).

${ }^{24}$ M. Tadic, N. Cukaric, V. Arsoski, and F. M. Peeters, Phys. Rev. B 84, 125307 (2011)

${ }^{25}$ P. Offermans, P. M. Koenraad, J. H. Wolter, D. Granados, J. M. García, V. M. Fomin, V. N. Gladilin, and J. T. Devreese, Appl. Phys. Lett. 87, 131902 (2005).

${ }^{26}$ D. Granados and J. M. García, Appl. Phys. Lett. 82, 2401 (2003).

${ }^{27}$ J. W. W. van Tilburg, R. E. Algra, W. G. G. Immink, M. Verheijen, E. P. A. M. Bakkers, and L. P. Kouwenhoven, Semicond. Sci. Technol. 25, 024011 (2010).

${ }^{28}$ F. Qian, Y. Li, S. Gradečak, H.-G. Park, Y. Dong, Y. Ding, Z. L. Wang, and C. M. Lieber, Nat. Mater. 7, 701 (2008).

${ }^{29}$ E. P. A. M. Bakkers and M. A. Verheijen, J. Am. Chem. Soc. 125, 3440 (2003).

${ }^{30}$ D. Loss and P. Goldbart, Phys. Rev. B 43, 13762 (1991).

${ }^{31}$ W.-C. Tan and J. C. Inkson, Phys. Rev. B 60, 5626 (1999).

${ }^{32}$ E. N. Bogachek, A. G. Scherbakov, and U. Landman, Phys. Rev. B 63, 115323 (2001).

${ }^{33}$ A. Aldea, V. Moldoveanu, M. Nită, A. Manolescu, V. Gudmundsson, and B. Tanatar, Phys. Rev. B 67, 035324 (2003).

${ }^{34}$ A. Messiah, Quantum Mechanics, Vol. II (3rd printing, NorthHolland, 1965), pp. 773-780.

${ }^{35}$ I. Kuljanishvili, C. Kayis, J. F. Harrison, C. Piermarocchi, T. A. Kaplan, S. H. Tessmer, L. N. Pfeiffer, and K. W. West, Nat. Phys. 4, 227 (2008).

${ }^{36}$ D. E. Perea, E. R. Hemesath, E. J. Schwalbach, J. L. Lensch-Falk, P. W. Voorhees, and L. J. Lauhon, Nat. Nanotechnol. 4, 315 (2009).

${ }^{37}$ E. Barnes, J. P. Kestner, N. T. T. Nguyen, and S. Das Sarma, Phys. Rev. B 84, 235309 (2011).

${ }^{38}$ T. Giamarchi, Quantum Physics in One Dimension (Clarendon, Oxford, 2003). 